Journal of Applied Pharmaceutical Science Vol. 3 (09), pp. 109-116, June, 2013

Available online at http://www.japsonline.com

DOI: $10.7324 /$ JAPS.2013.3920

ISSN 2231-3354 (cc) BY-NC-SA

\title{
Statistical Optimization of OlanzapineTernary Solid Dispersions with Pvp K 30 and Peg 20,000 by Response Surface Methodology
}

\author{
Anil Katharia*1,2, Ratendra Kumar ${ }^{3}$, Rajiv Sharma ${ }^{2}$, Yogendra Singh ${ }^{2}$, Uday Veer Singh Teotia ${ }^{1}$ \\ ${ }^{1}$ Shri venkateshwara university, gajraula distt. J.P Nagar ,Uttar Pradesh, India \\ ${ }^{2}$ Alembic pharmaceutical Ltd, Baddi, Himachal Pradesh, India \\ ${ }^{3}$ MIET, Meerut, Uttar Pradesh, India
}

\begin{tabular}{l}
\hline ARTICLE INFO \\
\hline Article history: \\
Received on: 20/06/2013 \\
Revised on: 07/07/2013 \\
Accepted on: 17/07/2013 \\
Available online: $30 / 09 / 2013$ \\
\hline Key words: \\
Olanzapine; Central \\
composite design; PEG \\
20000; PVP K30 and \\
dissolution enhancement.
\end{tabular}

\begin{abstract}
The aim of present study was to improve dissolution rate of olanzapine by means of solid dispersion using combination of hydrophilic polymer (PEG \& PVP) by using response surface design. Solid dispersion containing olanzapine were prepared using PEG 20000 \& PVP K 30 by melted fusion method. Response surface method was used for the optimization olanzapine solid dispersions. Amount of PEG 20000 and Amount of PVP K 30 were selected as the critical process parameters (Independent variable) whereas amount dissolved in 10 minute $\left(Q_{10)}\right.$ and amount dissolved in 45 minute $\left(Q_{45)}\right.$ were selected as critical quality attributes (dependent variables). Optimized solid dispersion batch was characterized using infrared spectroscopy, differential scanning calorimetry (DSC) and X-ray diffractometry (XRD).Dissolution studies indicated a significant improvement in dissolution of olanzapine when dispersed in PEG 20000 and PVP k 30. XRD and DSC study indicated amorphous form of prepared solid dispersions. On the basis of numerical optimization technique, PEG $20000\left(\mathrm{X}_{1}\right)$ and amount of PVP K $30\left(\mathrm{X}_{2}\right)$ were $11.20 \%$ and $14.53 \%$ in optimized solid dispersion. The observed responses were closed well with the predicted values. The response surface method is found to be robust and accurate for optimization of solid dispersion for increase in solubility and dissolution rate of olanzapine, coherent with the needs of poorly water soluble drugs.
\end{abstract}

\section{INTRODUCTION}

Olanzapine is an atypical antipsychotic. It is approved for the treatment of schizophrenia and bipolar disorder. Olanzapine is a BCS II drug (Poor solubility and high permeability)(Dixit, et al., 2011). The bioavailability of a BCS class II drug is rate-limited by its dissolution, so that even a small increase in dissolution rate sometimes results in a large increase in bioavailability (Lobenberg and Amidon, 2000). Therefore, an enhancement of the dissolution rate of the drug is thought to be a key factor for improving the bioavailability of BCS class II drugs. Crystal modification particle size reduction, self-emulsification, $\mathrm{pH}$ modification, and amorphization are considered to be effective for improving the dissolution behaviour of BCS class II drugs (Kaushal, et al., 2004) (Xia, et al., 2010, Tran, et al., 2010).

* Corresponding Author

Shri venkateshwara university, Department of Pharmaceutical Sciences Gajraula, J.P Nagar, Uttar Pradesh, India Pin code: 244235

Email : mailto_anilk@rediffmail.com, Phone No; +91-7696025571
Solid dispersions are one of the most successful strategies to improve drug release of poorly soluble drugs. These can be defined as molecular mixtures of poorly water soluble drugs in hydrophilic carriers, which present a drug release profile that is driven by the polymer properties (Chiou and Riegelman, 1971a, Das, et al.). The first drug whose rate and extent of absorption was significantly enhanced using the solid dispersion technique was sulfathiazole by Sekiguchi and Obi (Sekiguchi and Obi, 1961). Over the years, a variety of carriers have been used to prepare solid dispersion of olanzapine to improve dissolution rate such as; Croscarmellose sodium (Krishnamoorthy et al., 2011), Mannitol (Krishnamoorthy, et al., 2012), pregelatinised starch (PGS) and sodium starch glycollate (Krishnamoorthya, et al., 2011). Polyethylene glycols (PEG), polymers of ethylene oxide are widely used as vehicles for solid dispersions because of their low melting point, rapid solidification rate, capability of forming solid drug solutions, low toxicity and low costs (Betageri and Makarla, 1995, Chiou and Riegelman, 1971b). 
However, at higher drug concentrations, the drug is often present in the crystalline form within the PEG dispersion or it recrystallizes over time, resulting in unstable formulations with lower dissolution rates.

The combination of two hydrophilic polymeric carriers like PEG and PVP could improve the solubility as well as dissolution profiles of various poorly aqueous soluble drugs due to inhibition of drug recrystallization as well as a rapid solidification rate (Hasnain and Nayak, 2012, Bley, et al., 2010, Guedes, et al., 2011). Design of experiment could be used for the statistical optimization of pharmaceutical dosage forms. Central composite design is a type of response surface methodology that requires smaller number of experimental runs and is less time consuming than conventional formulation methods (Elbary, et al., December 2011, Akhgari, et al., 2005). The aim of this study is to improve dissolution rate of olanzapine by means of solid dispersion using combination of hydrophilic polymer (PEG \& PVP). The physicalchemical properties of olanzapine in solid dispersion was studied by differential scanning calorimeter ( DSC), X-ray diffraction (XRD) and Fourier transform infrared (FTIR).Dissolution profile of solid dispersion also studied. In order to accomplish this aim, computer-aided optimization techniques using two-factor, threelevel central composite design was used to determine the effect of two formulation factors, namely, the contents of PEG 20000 and PVP K 30 on the dissolution of drug and to statistically optimize the levels of these factors using multivariate analysis and response surface plots in order to incur the targeted dissolution rate for olanzapine.

\section{MATERIAL \& METHODS}

\section{Materials}

Olanzapine was kindly gifted by Micro labs Bangalore. PEG 20000 and PVP K 30 were obtained from SD Fine Chemicals Ltd. (Mumbai, India). All other materials and reagents were of analytical grade of purity.

\section{Preparation of solid dispersion}

Solid dispersion containing $12.9 \%$ olanzapine were prepared using PEG 20000 \& PVP K 30 by melted fusion method. The drug and polymer were heated until the polymer melt. The molten mixture was stirred until the drug was dissolved completely in the melt and a homogeneous solution was obtained. The solution was brought to solidification by quick cooling. It was kept in desiccators under vacuum for $24 \mathrm{hr}$. Then solid dispersion formulation was pulverized using mortar and pestle. The pulverized powder was classified using the sieve \# 60 .

\section{Experimental Design}

A central composite response surface design (DESIGN EXPERT 8.0.1 demo version software) was used for the optimization olanzapine solid dispersions. Amount of PEG 20000 (A) and Amount of PVP K 30 were selected as the independent variables whereas $Q_{10}$ (amount dissolved in 10 minute) and $Q_{45}$ (amount dissolved in 45 minute) were selected as dependent variables. Levels for two factors are presented in Table 1.

Table. 1: Central composite response surface design layout.

\begin{tabular}{|c|c|c|c|c|}
\hline \multirow{2}{*}{ Formulation } & \multicolumn{2}{|c|}{ Variables in coded Form } & \multirow{2}{*}{ Q10 } & \multirow[b]{2}{*}{ Q45 } \\
\hline & $\mathrm{X} 1(\%)$ & $\mathrm{X} 2(\%)$ & & \\
\hline SD 1 & -1.000 & -1.000 & 14.72 & 54.464 \\
\hline SD 2 & 1.00 & -1.000 & 16.14 & 62.464 \\
\hline SD 3 & -1.000 & 1.000 & 31.69 & 88.69 \\
\hline SD 4 & 1.000 & 1.000 & 36.21 & 89.42 \\
\hline SD 5 & -1.414 & 0.000 & 24.56 & 76.23 \\
\hline SD 6 & 1.414 & 0.000 & 29.07 & 82.49 \\
\hline SD 7 & 0.000 & -1.414 & 9.21 & 45.76 \\
\hline SD 8 & 0.000 & 1.414 & 34.76 & 89.32 \\
\hline SD 9 & 0.000 & 0.000 & 25.998 & 79.162 \\
\hline SD 10 & 11.20 & 14.53 & 24.70 & 76.13 \\
\hline \multirow[t]{2}{*}{ Coded Value } & \multicolumn{2}{|c|}{ Actual Value (\%) } & & \\
\hline & $\mathrm{X1}$ & $\mathrm{X} 2$ & & \\
\hline-1.414 & 7.93 & 7.93 & & \\
\hline-1.000 & 10.00 & 10.00 & & \\
\hline 0.000 & 15.00 & 15.00 & & \\
\hline 1.000 & 20.00 & 20.00 & & \\
\hline 1.414 & 22.07 & 22.07 & & \\
\hline
\end{tabular}

X1 indicates amount of PEG 20000 (\%); X2, amount of PVP K 30 (\%); Q 10 , amount dissolved in 10 minute; and $\mathrm{Q}_{45}$, amount dissolved in 45 minute. SD 10 used as checks point and optimized batch.

Different trial formulations of olanzapine solid dispersions were prepared according to the trial proposal of central composite response surface design. The prepared solid dispersions of Olanzapine were evaluated for dissolution study. The responses were analyzed using ANOVA and the individual response parameters were evaluated using $\mathrm{F}$ test and polynomial equation, generated for each response using multiple linear regression analysis (MLRA). The study design including investigated factors and responses is shown in Table 1 . The optimized formulation was prepared which have the $\mathrm{Q}_{10}$ in range $20-25 \%$ and $\mathrm{Q}_{45}$ in range $75-80 \%$. Constraints for responses and factors are shown in Table 2. By utilizing the software, we got one solution for optimized formulation. The optimized formulation was prepared and evaluated for $\mathrm{Q}_{10}$ and $\mathrm{Q}_{45}$. Observe response value of the optimized formulation was compared with predicted value. The optimised batch(s) was further investigated by DSC, XRD, and FTIR.

Table. 2: Optimization of olanzapine solid dispersion.

\begin{tabular}{|c|c|c|c|}
\hline \multicolumn{4}{|c|}{ Constraints } \\
\hline Name & Goal & Lower limit & Upper Limit \\
\hline PEG 20000 & In range & -1.414 & 1.414 \\
\hline PVP K 30 & In range & -1.414 & 1.414 \\
\hline $\mathrm{Q}_{10}(\%)$ & In range & 20 & 25 \\
\hline $\mathrm{Q}_{45}(\%)$ & In range & 75 & 80 \\
\hline \multicolumn{4}{|c|}{ SOLUTION (SD 10) } \\
\hline PEG 20000 & PVP K 30 & $\mathrm{Q}_{10}(\%)$ & Desirability \\
\hline 11.20 & 14.53 & 24.70 & 1.000 \\
\hline
\end{tabular}

\section{In vitro dissolution study}

Drug dissolution studies was carried out using USP dissolution apparatus 2 using a paddle at a speed of $100 \mathrm{rpm}$ with $900 \mathrm{~mL}$ of Phosphate buffer $\mathrm{pH}$ 7.4. as dissolution medium at 
$37^{0} \mathrm{C}$. Solid dispersion powders containing $50 \mathrm{mg}$ of olanzapine were dispersed on the surface of the dissolution medium and the time was recorded. At intervals, $5 \mathrm{~mL}$ samples were withdrawn through a filter.

All the readings were blanked with same media as was used in the dissolution study. The olanzapine content was measured by HPLC method and the percentage of drug released was calculated using calibration curves.

\section{Drug release kinetic}

The release from the different SD formulations was determined by curve fitting method. Data obtained from in vitro release studies were fitted to various kinetic equations.

Zero order Model: $Q_{t}=Q_{0}+k_{0} t$, where $Q_{\mathrm{t}}$ is the amount of drug dissolved in time $t, Q_{0}$ is the initial amount of drug in the solution and $K_{0}$ is the zero order release constant.

First order Model: $\ln \left(Q_{\infty}-Q_{t}\right)=\ln Q_{0}+k t$, where $Q_{\mathrm{t}}$ is the amount of drug dissolved in time $t, Q_{0}$ is the initial amount of drug in the solution, $\mathrm{Q}_{\infty}$ is the amount release in time $\infty(100 \%$ drug release) and $K$ is the first order release constant.

Korsmeyer-Peppas Model (power law): $\frac{Q_{t}}{Q_{\infty}}=k_{k} t^{n}$, or $\log Q_{t}=$ $\log k_{k}+n \log t$ where $Q_{\mathrm{t}}$ is the amount of drug dissolved in time $t, \mathrm{Q}_{\infty}$ is the amount release in time $\infty, \mathrm{k}_{\mathrm{k}}$ is the rate constant and $\mathrm{n}$ is the diffusional exponent, this indicates the drug release mechanism.

\section{$X$-ray diffraction}

Powder X-ray diffraction patterns were obtained with a diffractometer (Geigerflex, RAD-IB, Rigaku, Tokyo). The operating conditions were as follows: target, $\mathrm{Cu}$; filter, $\mathrm{Ni}$; voltage, $40 \mathrm{kV}$; current, $20 \mathrm{~mA}$ and scanning speed, $2 \theta=4^{\circ}$ :min. Physical mixtures, as control of the solid dispersion, were prepared by simply mixing the powdered olanzapine and polymers at the same composition ratios as those of the solid dispersions.

\section{Differential scanning calorimetry (DSC)}

DSC studies of Olanzapine and optimized solid dispersion batch were conducted using a Perkin Elmer DSC-4 differential scanning calorimeter using aluminium sample pans for volatiles. Samples (about $5 \mathrm{mg}$ ) were heated at $10{ }^{\circ} \mathrm{C} / \mathrm{min}$ using nitrogen as the purging gas.

\section{Fourier transform infrared spectroscopy (FTIR)}

An approximately minimum quantity (about $1 \mathrm{mg}$ ) of sample was thoroughly blended with adequate quantity of IR grade KBR (about $5 \mathrm{mg}$ ) in a mortar. The mix was then made into thin films on a sample plate using a hand operated compression lever. The samples were then analyzed in a Perkin Elmer Model 1330 double beam IR spectrometer using $\mathrm{KBr}$ film as negative control (blank).

\section{RESULT AND DISCUSSION}

A total of 9 trial formulations of olanzapine solid dispersion were proposed by central composite response surface design for two independent variables: Amount of PEG 20000 and amount of PVP K 30 which were varied at three different levels. All the batches of solid dispersion were evaluated for its amount dissolved in 10 minute $\left(\mathrm{Q}_{10}\right)$ and amount dissolved in 45 minute $\left(\mathrm{Q}_{45}\right)$. The dependent variables obtained at three levels of the 2 independent variables (X1 and $\mathrm{X} 2$ ) was subjected to multiple regression to yield a interactive and polynomial equation. The polynomial equation generated by this experimental design was as follows:

$Y_{i}=b_{0}+b_{1} X_{1}+b_{2} X_{2}+b_{12} X_{1} X_{2}+b_{11} X_{1}^{2}+b_{22} X_{2}^{2}$

Where $\mathrm{y}_{\mathrm{i}}$ is the dependent variable, $\mathrm{b}_{0}$ is the arithmetic mean response of the 9 runs; and $b_{1}$ and $b_{2}$ are the estimated coefficients for the independent factors $X_{1}$ and $X_{2}$, respectively. The main effects $\left(X_{1}\right.$ and $\left.X_{2}\right)$ represent the average result of changing one factor at a time from its low to high value. The interaction term $\left(\mathrm{X}_{1} \mathrm{X}_{2}\right)$ shows how the response changes when 2 factors are simultaneously changed. The polynomial terms $\left(\mathrm{X}_{1}^{2}\right.$ and $\left.\mathrm{X}_{2}^{2}\right)$ are including investigating nonlinearity.

The $\mathrm{Q}_{10}$ and $\mathrm{Q}_{45}$ for the 9 trial batches (SD 1 to SD 9) showed a wide variation from 9.21 to $36.21 \%$ and from 45.76 to $89.42 \%$ respectively(Table 1$)$. The observation show that the Q 10 and Q 45 strongly depends upon the selected independent variables. The model (full and reduced) relating the responses, $Q$ 10 and Q 45 to the transformed factor are shown in table 4.The result of the analysis of variance (ANOVA) for the model simplification by eliminating non significant terms $(P>0.05)$ were shown in table 5. For both Q 10 and Q 45 term $\mathrm{X}_{1}^{2}$ was found to be insignificant ( $p$ value $>0.05$ ), hence this was omitted from the full model to generate the reduced models. The $\mathrm{F}$ value in the ANOVA table was the ratio of model mean square (MS) to the appropriate error (i.e. residual) mean square. The larger the $\mathrm{F}$ value and the more likely that the variance contributed by the model was significantly larger than random error. The model F-value and high $\mathrm{R}$ square values suggested that these models were significant.

The results of multiple linear regression analysis reveal that both the coefficient $b_{1}$ and $b_{2}$ bear a positive sign for both $Q$ 10 and $\mathrm{Q}$ 45.Therefore, increasing the amount of either PEG 20000 or PVP K 30 is expected to increase the Q 10 and Q 45. Three-dimensional response surface plots and corresponding contour plots to study the effects of the independent variables (factors) on each dependent variable (response) were presented in Figure 1 and 2.

A numerical optimization technique based on the desirability approaches was adopted to achieve new optimized solid dispersion (SD 10) which was also used as the check point. For evaluation the optimization capability of response surface factorial design. The variable settling used for the formulation of optimized solid dispersion were amount of PEG $20000\left(X_{1}\right)$ and amount of PVP K $30\left(\mathrm{X}_{2}\right)$ were $11.20 \%$ and $14.53 \%$ (Fig.3 and 
table 2). The optimized formulations were prepared with the optimized amount of independent variables. Observed values were found similar to predicted values in SD 10. Thus, we can conclude that the statistical model was mathematically valid.

\section{In vitro dissolution study}

Drug release from the different solid dispersion formulation and pure powdered drug is shown in figure 4 . Compared with the pure olanzapine, the dissolution of olanzapine increases by PEG-PVP solid dispersion. The increase in dissolution of olanzapine with PEG-PVP could be attributed by the reduction in crystallinety of olanzapine. All the release profile shows two different phases of drug release. An initial rapid release phase followed by a slower one. Same result was reported by Omaima A et al (2006) for rofecoxib solid dispersion(Omaima A Sammour, et al., 2006).

\section{Drug release kinetic}

The drug release data were analyzed using the zero-order and first-order equations to determine the drug release kinetics from the solid dispersion formulation. The regression coefficient $\left(\mathrm{r}^{2}\right)$ data based on kinetic analysis using various release models are listed in Table 5. To find out the mechanism of drug release first $60 \%$ drug release data was fitted in Korsmeyer-Peppas model, in which log cumulative percentage of drug release was plotted against time.

The regression coefficients (table 5) obtained for zero order kinetics were found to be higher $\left(r^{2}=0.9862\right.$ to 0.9994) when compared with those of first order kinetics $\left(r^{2}=0.9387\right.$ to 0.0 .998$)$, indicating that drug release independent of concentration from all formulation. All the formulation shows good linearity $\left(\mathrm{r}^{2}=0.9890\right.$ to 0.9986$)$, with the slope $(n)$ values 0.5896 to 1.014 , indicating that release mechanism was anomalous non-Fickian or anomalous release $(0.5<n<1.0)$, which indicates that the drug release occurred through diffusion in the hydrated matrix and polymer relaxation.

\section{Powder X-Ray diffraction study.}

The $\mathrm{x}$-ray powder differtometry (XRD) study of olanzapine and optimized solid dispersion is done in the following manners. The angular range is 5 to $50^{\circ}, 2 \theta$ counts are accumulated for $1 \mathrm{sec}$ at each step. A typical $\mathrm{x}$ ray diffraction of olanzapine \& solid dispersion are shown in figure 5. The pure olanzapine XRD showed numerous sharp narrow and intense peaks indicating its high crystallnity. Solid dispersion of olanzapine did not show the characteristic peak, indicates reduction in crystalline and phase transition from crystalline to amorphous from in the solid dispersion sample.

\section{Differential scanning calorimetry (DSC)}

DSC curve obtained for pure olanzapine and solid dispersion prepared by melting method. Pure olanzapine showed a melting endothern $197.5^{\circ} \mathrm{C}$. The DCS of solid dispersion showed melting point for the PEG around $59^{\circ} \mathrm{C}$ with no endothermic peak corresponding to the Olanzapine (figure 6). The absence of peak at temp corresponding to the melting of the drug could potentially be assigned to the solubility and distribution of the drug within the polymer matrix resulting in the conversion of crystalline drug form into amorphous form.

\section{Fourier transforms infrared spectroscopy (FTIR)}

The IR spectra for olanzapine was characterised by sharp transition occurring at 1033, 2225, 1559 and $745 \mathrm{~cm}^{-1}$ corresponding to the bond stretching associated with $\mathrm{O}-\mathrm{H}$ bending, $\mathrm{N}-\mathrm{H}$ stretching, $\mathrm{C}=\mathrm{N}$ stretching and $\mathrm{C}-\mathrm{S}$ bending respectively. Analysis of the spectra for the solid dispersion of olanzapine (figure 7) did not reveal any changes for the specific absorption bands for the drug suggesting a lack of interaction with carrier moieties. It was also noticed that the significant peaks of pure drug were found to be less sharpness and more broadness in the solid dispersion sample. These findings clearly prove the reduction of crystallinity in the drug molecule present in samples (AYALA, et al., 2006, AYALA, 2007, Krishnamoorthy, et al., 2012).
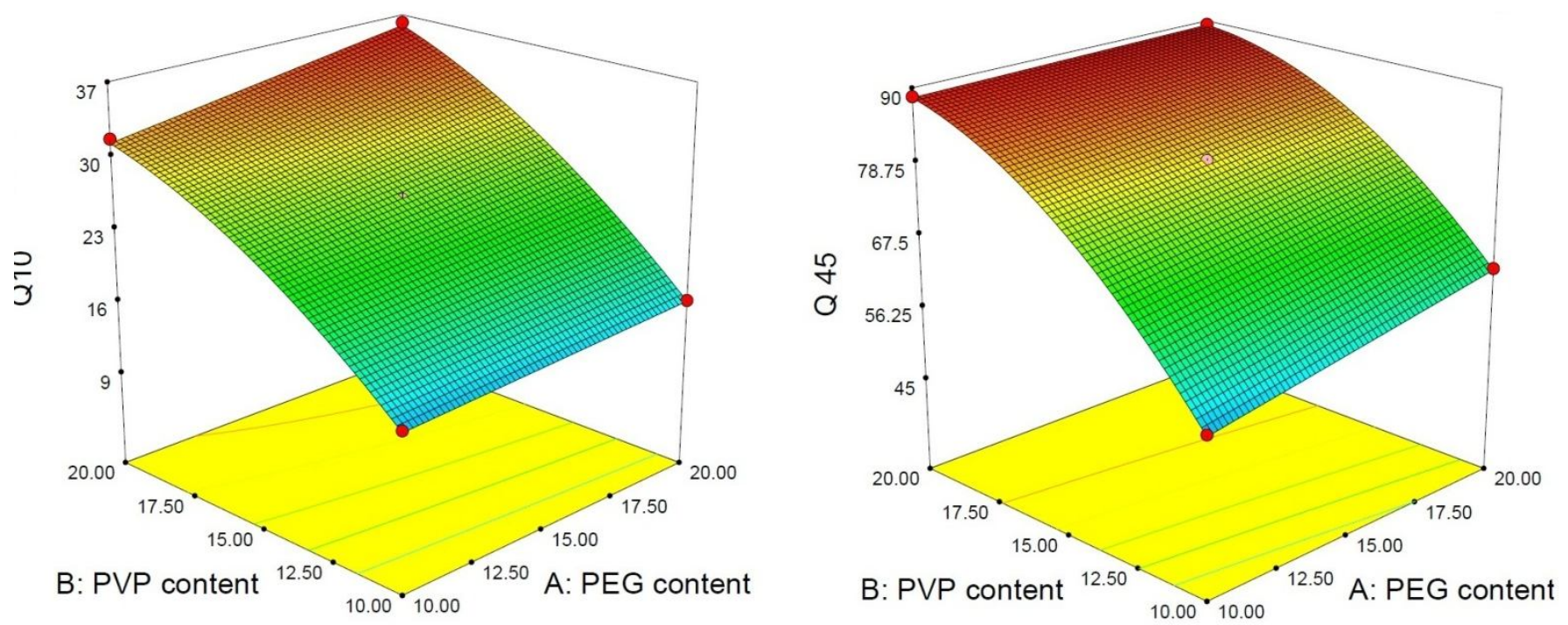

Fig. 1: 3 D response surface plot showed the effect of amount of PEG 20000 (X1) and amount of PVP K 30 (X2) on Q 10 (amount release at 10 minute) and Q 45 (amount release at 45 minute). 
Table. 3: Summary of results of regression analysis.

\begin{tabular}{|c|c|c|c|c|c|c|}
\hline \multicolumn{7}{|c|}{ For $Q_{10}$} \\
\hline Response & $\mathbf{b}_{0}$ & $\mathbf{b}_{1}$ & $\mathbf{b}_{2}$ & $b_{12}$ & $\mathbf{b}_{11}$ & $b_{22}$ \\
\hline FM & 26.0 & 1.54 & 9.15 & 0.78 & 0.48 & -1.93 \\
\hline$P$ value & - & 0.0008 & $<0.0001$ & 0.0159 & 0.0793 & 0.0018 \\
\hline RM & 26.70 & 1.54 & 9.15 & 0.78 & - & -2.24 \\
\hline$P$ value & - & 0.0009 & $<0.0001$ & 0.0345 & - & 0.0005 \\
\hline \multicolumn{7}{|c|}{ For $Q_{45}$} \\
\hline FM & 79.16 & 2.20 & 15.35 & -0.1 .82 & 0.18 & -5.73 \\
\hline$P$ value & - & 0.0002 & $<0.0001$ & 0.0009 & 0.3435 & $<0.0001$ \\
\hline $\mathrm{RM}$ & 79.42 & 2.20 & 15.35 & -0.1 .82 & - & -5.85 \\
\hline$P$ value & - & $<0.0001$ & $<0.0001$ & 0.0002 & - & $<0.0001$ \\
\hline
\end{tabular}

FM indicates Full model and RM, Reduce Model.

Table. 4: Result of Analysis of variance (ANOVA).

\begin{tabular}{|c|c|c|c|c|c|}
\hline \multicolumn{6}{|c|}{ For $\mathrm{Q}_{10}$} \\
\hline Regression & Df & SS & MS & $\mathbf{F}$ & $\mathbf{R}^{2}$ \\
\hline $\mathrm{FM}$ & 5 & 716 & 143 & 1456.23 & 0.9996 \\
\hline RM & 4 & 715.19 & 178.80 & 738.80 & 0.9986 \\
\hline \multicolumn{6}{|c|}{ Residual } \\
\hline FM & 3 & 0.29 & 0.098 & & \\
\hline RM & 4 & 0.97 & 0.24 & & \\
\hline \multicolumn{6}{|c|}{ For $\mathrm{Q}_{45}$} \\
\hline FM & 5 & 2103.55 & 420.71 & 5852.91 & 0.9999 \\
\hline RM & 4 & 2103.46 & 525.86 & 6870.95 & 0.9999 \\
\hline \multicolumn{6}{|c|}{ Residual } \\
\hline FM & 3 & 0.22 & 0.072 & & \\
\hline $\mathrm{RM}$ & 4 & 0.31 & 0.077 & & \\
\hline
\end{tabular}

DF indicates: degrees of freedom; SS, sum of squares; $M S$, mean of squares; $F$, ischer's ratio; $R^{2}$, regression coefficient

Table .5: Regression coefficient for different drug release kinetic model.

\begin{tabular}{|c|c|c|c|c|}
\hline \multirow[t]{2}{*}{ Formulation } & \multirow{2}{*}{$\begin{array}{c}\text { Zero Order Kinetic } \\
\mathbf{r}^{2}\end{array}$} & \multirow{2}{*}{$\begin{array}{c}\text { First order Kinetic } \\
\mathbf{r}^{2}\end{array}$} & \multicolumn{2}{|c|}{ Korsmeyer-Peppas Model } \\
\hline & & & $\mathbf{r}^{2}$ & $\mathbf{n}$ \\
\hline SD 1 & 0.998 & 0.983 & 0.9968 & 0.94 \\
\hline SD 2 & 0.9973 & 0.993 & 0.9979 & 0.97 \\
\hline SD 3 & 0.9971 & 0.9431 & 0.9986 & 0.65 \\
\hline $\mathrm{SD} 4$ & 0.9958 & 0.9425 & 0.9969 & 0.55 \\
\hline SD 5 & 0.9969 & 0.9708 & 0.9980 & 0.76 \\
\hline SD 6 & 0.9943 & 0.9666 & 0.9964 & 0.73 \\
\hline SD 7 & 0.9994 & 0.9908 & 0.9982 & 1.01 \\
\hline SD 8 & 0.9868 & 0.9387 & 0.9890 & 0.71 \\
\hline SD 9 & 0.9942 & 0.9711 & 0.9957 & 0.80 \\
\hline
\end{tabular}

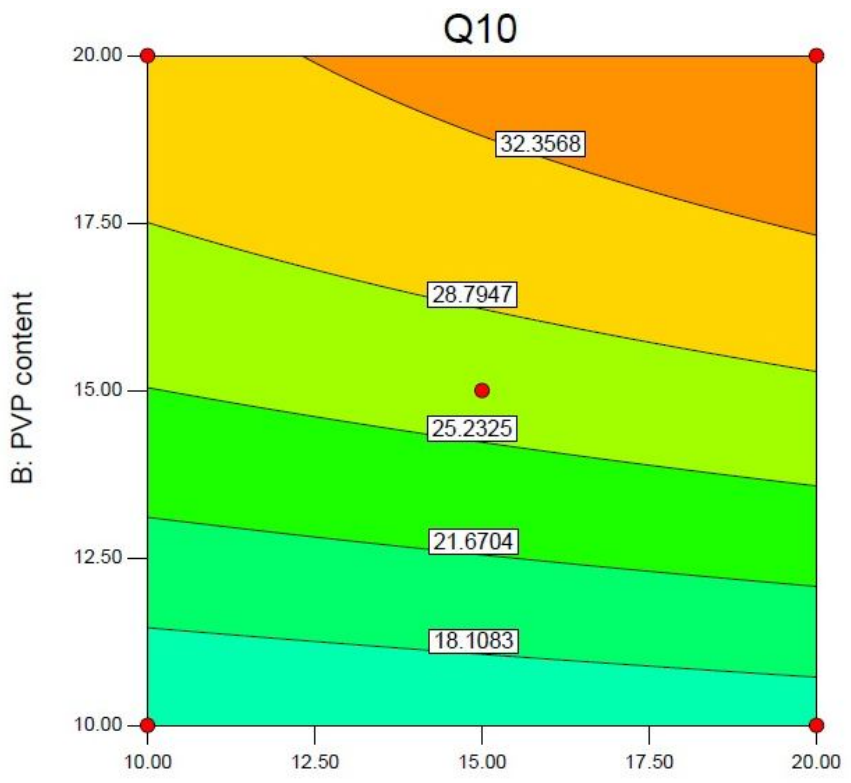

A: PEG content

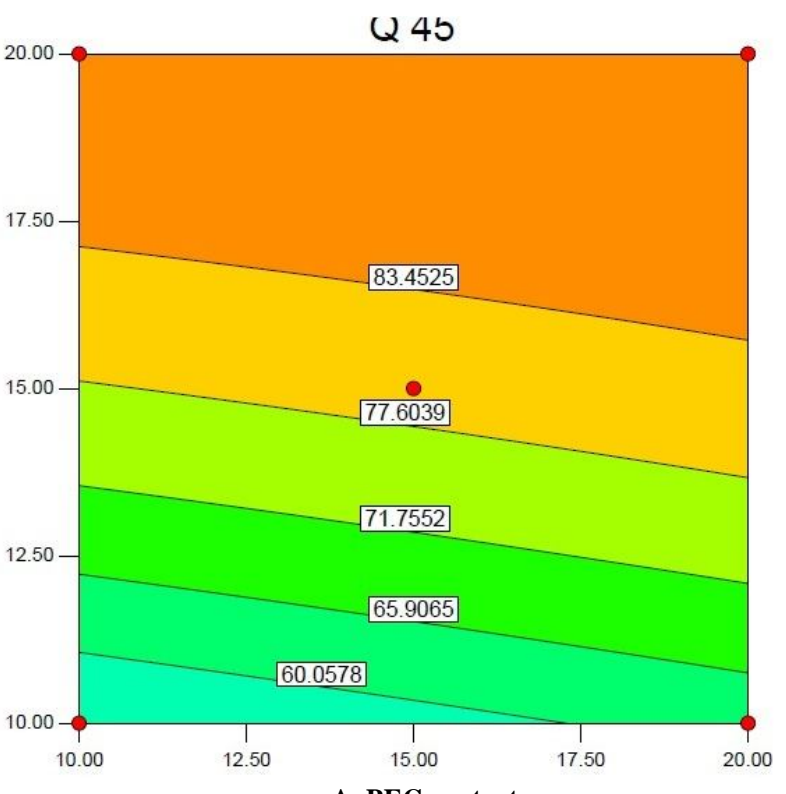

A: PEG content

Fig. 2: Contour surface plot showed the effect of amount of PEG 20000 (X1) and amount of PVP K 30 (X2) on Q 10 (amount release at 10 minute) and Q 45 (amount release at 45 minute). 

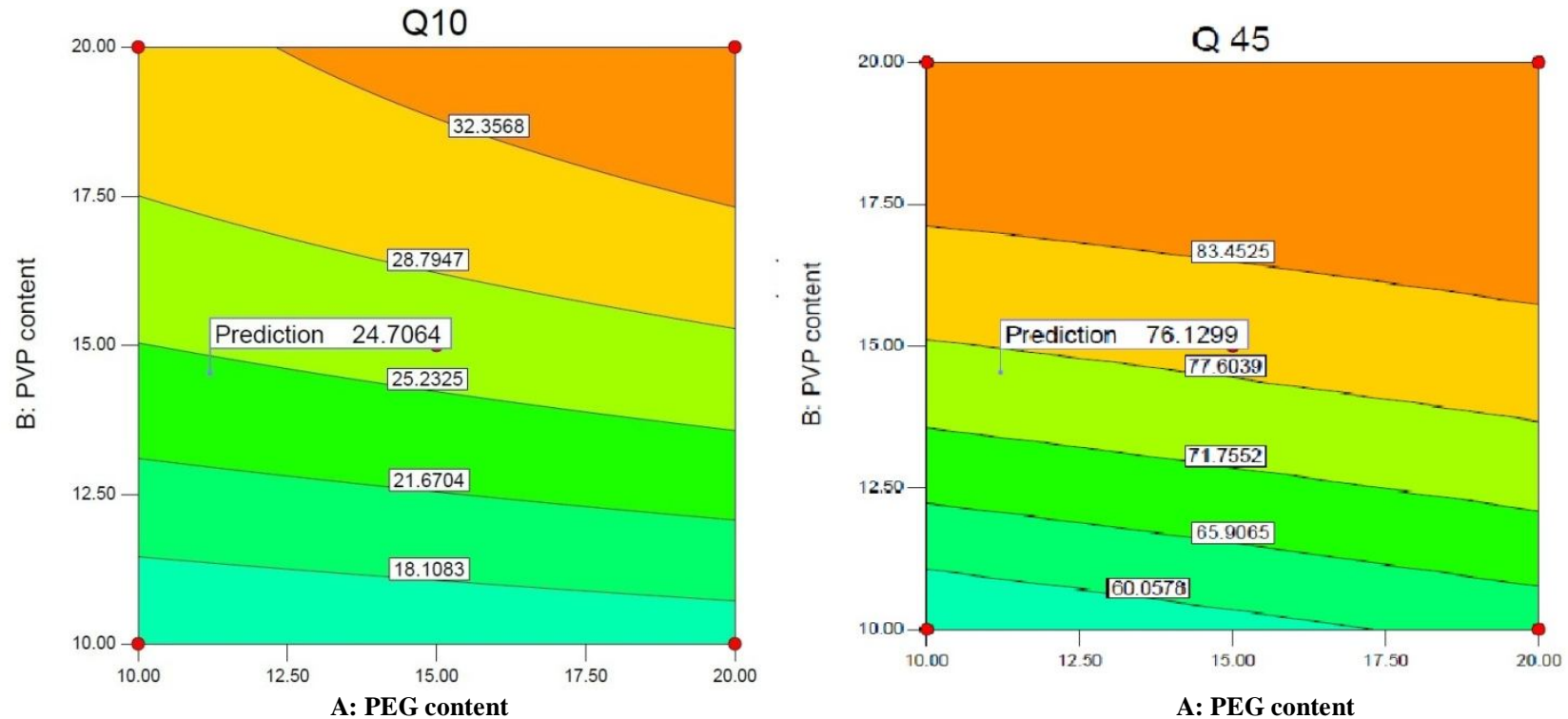

Fig. 3: Contour response surface Q 10 (amount release at 10 minute) and Q 45 (amount release at 45 minute) prediction plot.
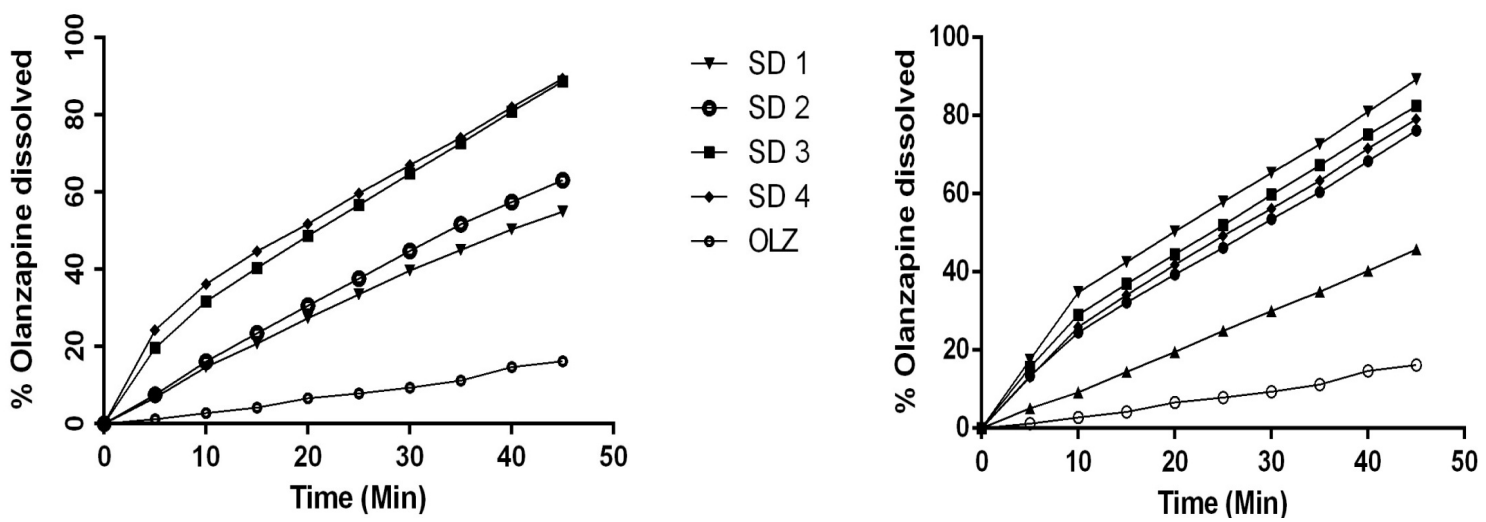

$\rightarrow$ SD 5

$\rightarrow$ SD 6

+ SD 7

$\rightarrow$ SD 8

$\rightarrow$ SD 9

$\rightarrow$ OLZ

Fig. 4: Drug release from the different solid dispersion formulation and pure powdered drug.
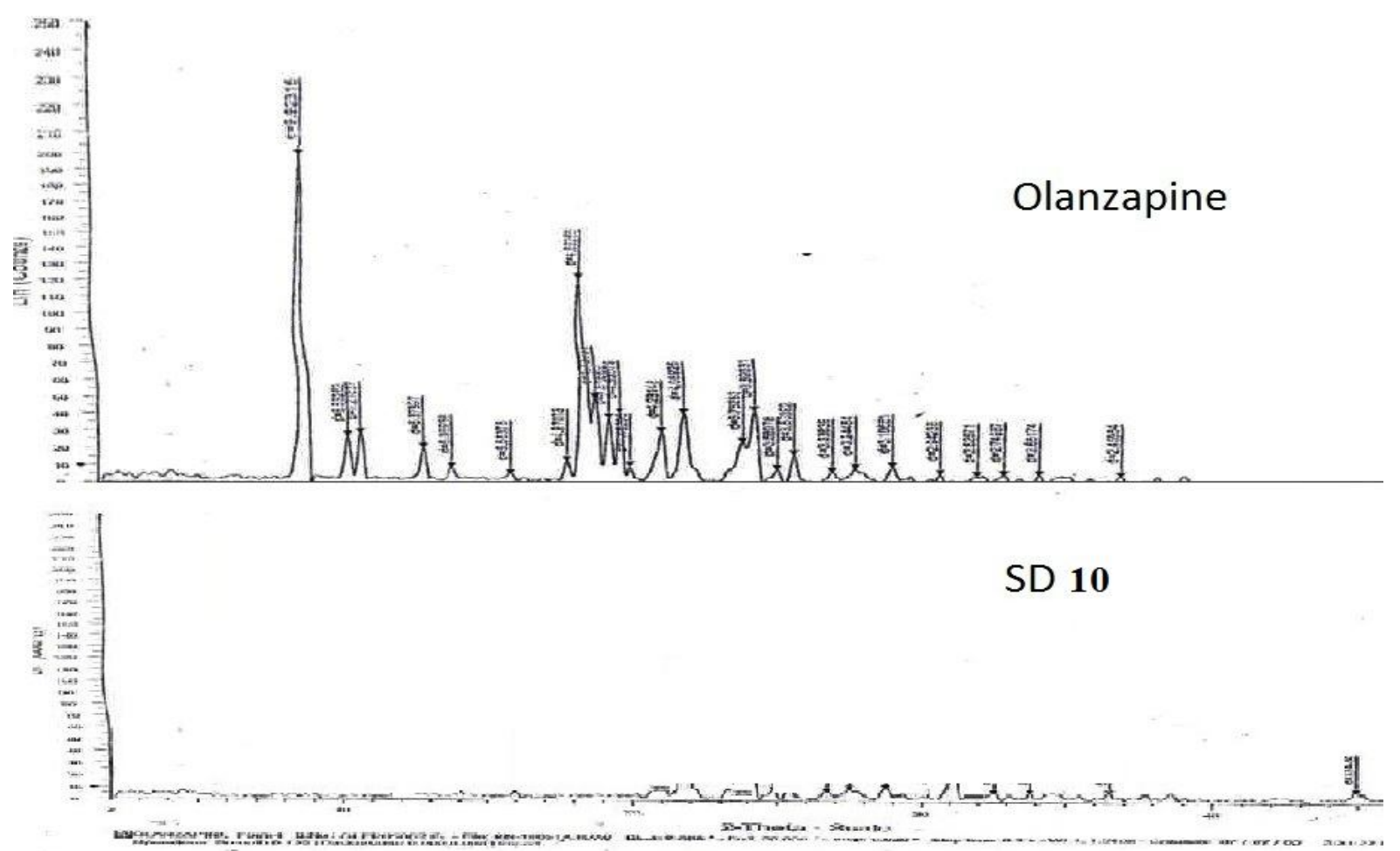

Fig. 5: X-ray powder differtometry (XRD) study of olanzapine and optimized solid dispersion (SD 10). 


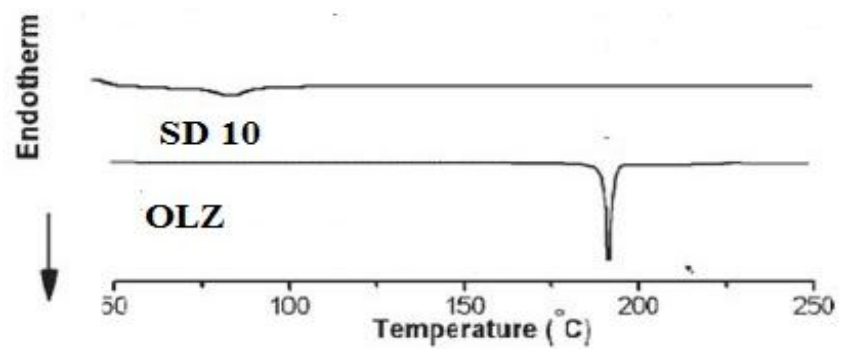

Fig. 6: DSC study of olanzapine and optimized solid dispersion (SD 10).

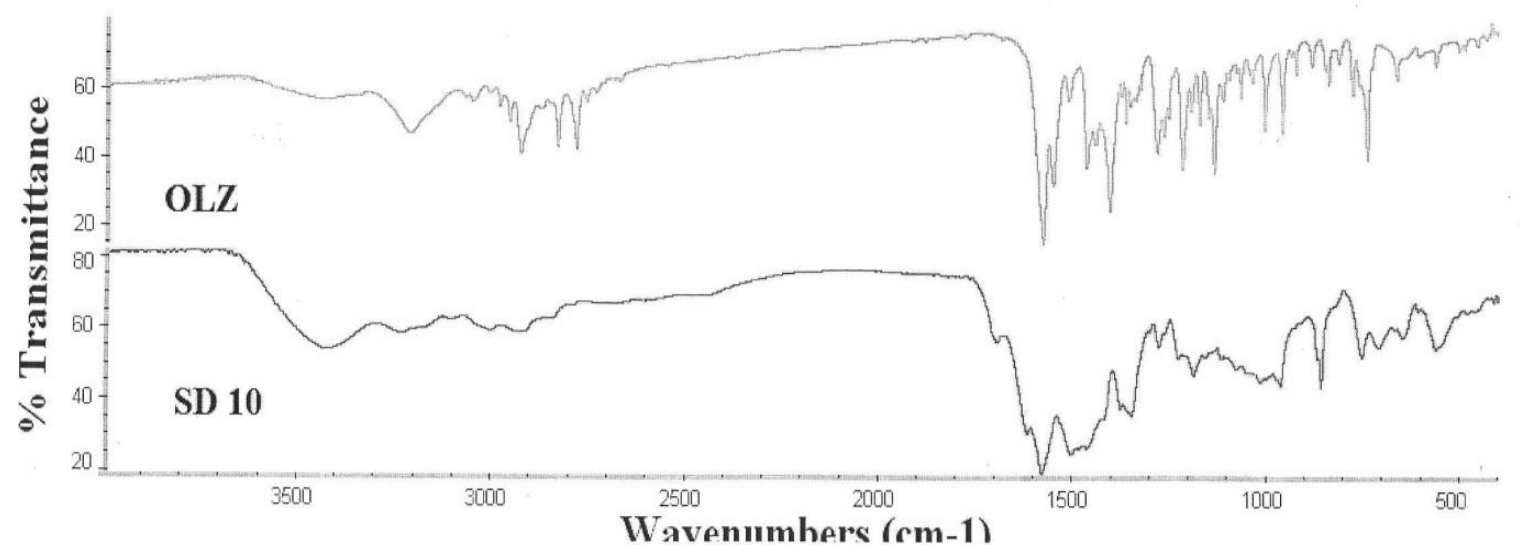

Fig. 7: FTIR study of olanzapine and optimized solid dispersion (SD 10).

\section{CONCLUSION}

The dissolution of olanzapine may be improved by the formation of solid dispersions with PEG 20000 and PVP K30 blends as carrier by the melting method. The central composite design was used to optimize the formulation. The threedimensional response surface plots and corresponding contour plots relating $Y 1\left(\mathrm{Q}_{10}\right)$ and $\mathrm{Y} 2\left(\mathrm{Q}_{45}\right)$ indicated the synergistic effect on both responses with the increase in X 1 (amount of PEG 20000) value and X 2 (amount of PVP K30) value. The optimized formulation with PEG 20000 (11.20 \%) and PVP K $30(14.53 \%)$ showed $24.7 \%$ Q10 and 76.132\% Q45). Thus the formulation can be considered as one of the promising tool for improvement of dissolution and solubility of olanzapine.

\section{REFRENCES}

Akhgari, A., Garekani, H. A., Sadeghi, F. and Azimaie, M.Statistical optimization of indomethacin pellets coated with pHdependent methacrylic polymers for possible colonic drug deivery. Int $J$ Pharm , 2005; 305:22-30.

Ayala, A. P.Polymorphism in drugs investigated by low wave number Raman scattering. Vib. Spectrosc., 2007; 45:112-116.

Ayala, A. P., Siesler, H. W., Boese, R., Hoffmann, G. G., Polla, G. I. and Vega, D. R. Solid state characterization of olanzapine polymorphs using vibrational spectroscopy. Int. J. Pharm., 2006; 326:6979.

Betageri, G. V. and Makarla, K. R.Enhancement of dissolution of glyburide by solid dispersion and lyophilization techniques. Int. J. Pharm., 1995; 126:155-166.
Bley, H., Fussnegger, B. and Bodmeier, R.Characterization and stability of solid dispersions based on PEG/polymer blends. International Journal of Pharmaceutics 2010; 390:165-173.

Chiou, W. L. and Riegelman, S.Pharmaceutical applications of solid dispersion systems. J.Pharm. Sci., 1971a; 60:1281-1302.

Chiou, W. L. and Riegelman, S.Pharmaceutical applications of solid dispersions. J. Pharm. Sci., 1971b; 60:1281-1302.

Das, S. K., Roy, S., Kalimuthu, Y., Khanam, J. and Nanda, A.Solid Dispersions : An Approach to Enhance the Bioavailability of Poorly Water-Soluble Drugs. International Journal of Pharmacology and Pharmaceutical Technology, 1:2277 - 3436.

Dixit, M., Kini, A. G. and Kulkarni, P. K.Enhancing the aqueous solubility and dissolution of olanzapine using freeze-drying. Brazilian Journal of Pharmaceutical Sciences, 2011; 47:743-749.

Elbary, A. A., Aboelwafa, A. A. and Sharabi, I. M. A.Once Daily, High-Dose Mesalazine Controlled-Release Tablet for Colonic Delivery: Optimization of Formulation Variables Using Box-Behnken Design. AAPS PharmSciTech, 2011; 12:1454-1464.

Guedes, F. L., Oliveira, B. G. D., Hernandes, M. Z., Simone, C. a. D., Veiga, F. J. B., Lima, M. D. C. a. D., Pitta, I. R., Galdino, S. L. and Neto, P. J. R.Solid Dispersions of Imidazolidinedione by PEG and PVP Polymers with Potential Antischistosomal Activities. AAPS PharmSciTech, 2011; 12:401-410.

Hasnain, M. S. and Nayak, A. K.Solubility And Dissolution Enhancement Of Ibuprofen By Solid Dispersion Technique Using Peg 6000-Pvp K 30 Combination Carrier. Chemistry: Bulgarian Journal of Science Education, 2012; 21:118-132.

Kaushal, A. M., Gupta, P. and Bansal, A. K.Amorphous drug delivery systems: molecular aspects, design, and performance. Critical Reviews in Therapeutic Drug Carrier System, 2004; 21:133-193.

Krishnamoorthy, V., Suchandrasen and Prasad, V. P. R.Physicochemical Characterization and In-Vitro Dissolution Behavior of Olanzapine - Croscarmellose Sodium Solid Dispersions. 
International Journal of Pharmacy and Pharmaceutical Sciences, 2011; 3:387-396.

Krishnamoorthy, V., Suchandrasen and Prasad, V. P. R.Physicochemical characterization and in vitro dissolution behavior of olanzapine-mannitol solid dispersions. Brazilian Journal of Pharmaceutical Sciences, 2012; 48:244-255.

Krishnamoorthya, V., Nagalingama, A., Prasadb, V. P. R., Parameshwarana, S., Georgea, N. and Kaliyana, P.Characterization of Olanzapine-Solid Dispersions. Iranian Journal of Pharmaceutical Research, 2011; 10:13-24.

Lobenberg, R. and Amidon, G. L.Modern bioavailability, bioequivalence and bio- pharmaceutics classification system . New scientific approaches to international regulatory standards. European Journal of Pharmaceutics and Biopharmaceutics 2000; 50:3-12.

Omaima a Sammour, Mohammed a Hammad, Nagia a Megrab and Zidan, A. S.Formulation and optimization of mouth dissolve tablets containing rofecoxib solid dispersion. AAPS PharmSciTech, 2006; 7:E 55.

Sekiguchi, K. and Obi, N.Studies on absorption 2 of eutectic mixture" I. A comparison of the behavior of eutectic mixture of sulfathiazole and that of ordinary sulfathiazole in man. Chem.Pharm. Bull., 1961; 9:866-872.

Tran, P. H., Tran, T. T., Lee, K. H., Kim, D. J. and Lee, B. J.Dissolution-modulating mechanism of $\mathrm{pH}$ modifiers in solid dispersion containing weakly acidic or basic drugs with poor water solubility. Expert Opinion on Drug Delivery, 2010; 7:647 -661.

Xia, D., Cui, F., Piao, H., Cun, D., Jiang, J., Ouyang, M. and Quan, P.Effect of crys- tal size on the in vitro dissolution and oral absorption of nitrendipine in rats. Pharmaceutical Research, 2010; 27:1965-1976.

\section{How to cite this article:}

Anil Katharia, Ratendra Kumar, Rajiv Sharma, Yogendra Singh, Uday Veer Singh Teotia., Statistical Optimization of Olanzapine Ternary Solid Dispersions with Pvp K 30 and Peg 20,000 by Response Surface Methodology. J App Pharm Sci, 2013; 3 (09): 109-116. 